Sociologie et sociétés

\title{
Points de vue logicistes sur les méthodologies en sciences
} sociales

\section{Logical Perspectives on Methodologies in the Social Sciences}

\section{Jean-Claude GARDIN}

Volume 25, numéro 2, automne 1993

La construction des données

URI : https://id.erudit.org/iderudit/001281ar

DOI : https://doi.org/10.7202/001281ar

Aller au sommaire du numéro

Éditeur(s)

Les Presses de l'Université de Montréal

ISSN

0038-030X (imprimé)

1492-1375 (numérique)

Découvrir la revue

Citer cet article

GARDIN, J.-C. (1993). Points de vue logicistes sur les méthodologies en sciences sociales. Sociologie et sociétés, 25(2), 11-22. https://doi.org/10.7202/001281ar
Résumé de l'article

Dans une perspective logiciste, les textes interprétatifs des sciences sociales sont justiciables d'une réécriture à la manière d'un calcul, qui fait ressortir les données et les opérations constitutives du raisonnement. Les recherches menées dans cette voie, en archéologie notamment, éclairent d'un jour nouveau les mécanismes et les fondements des constructions propres aux sciences de l'homme. Les leçons tirées portent sur le statut de l'explication dans ces disciplines, sur la part du sujet dans la conception et dans la validation de nos théories, sur l'intérêt d'une épistémologie pratique où ces théories sont jaugées selon des critères de valeur clairement déclarés, sur la neutralité d'une telle épistémologie à l'égard des méthodes des sciences sociales; enfin et surtout, sur la fragilité d'un entre-deux où celles-ci tentent sans relâche de se placer, entre science et littérature ou sens commun. 


\section{Points de vue logicistes sur les méthodologies en sciences sociales}

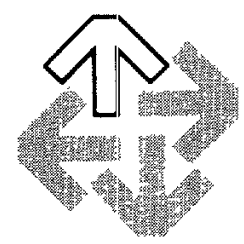

JEAN-CLAUDE GARDIN

Les notes d'intention que les éditeurs de ce numéro ont bien voulu me communiquer lorsqu'ils m'ont fait l'honneur de m'inviter à y participer rejoignent curieusement les termes d'un appel d'offres diffusé par la Délégation Générale à la Recherche Scientifique et Teclnnique, il y a 20 ans, en France, au titre de ce qu'on appelait dans le jargon de l'époque une Action Complémentaire Coordonnée, sur le thème "Informatique et Sciences humaines ». La reproduction de ce document, in fine, sera peut-être mon apport le plus utile à l'entreprise présente : elle lui fournit des racines que l'on peut qualifier d'anciennes, profondes par conséquent, et confirme la permanence des questions soulevées. Pour le reste de ma contribution, je crains qu'elle n'intéresse guère «les méthodologies des sciences sociales " auxquelles ce recueil est consacré. Deux raisons à cela, l'une simple, l'autre plus contournée : mon domaine de recherche est l'archéologie qui ne passe pas pour une discipline des sciences sociales. En second lieu, les questions de méthode qui m'occupent, ou les réponses que je leur donne, n'ont pas eu jusqu'ici beaucoup d'écho en sociologie. Le phénomène a certainement des causes sérieuses, qui le justifient; $j$ 'en retire des doutes sur la pertinence des notes qui suivent pour les lecteurs de ce numéro. Les deux coéditeurs, en revanche, semblent avoir à ce sujet une opinion différente; je me rends à leur avis, mais conserve mes craintes.

La principale est la crainte de lasser : les travaux et les idées que l'on m'invite à présenter n'ont plus rien d'inédit. On comprendra que je me borne à évoquer les premiers en quelques lignes, accompagnées des références voulues. Pour les secondes, je tenterai de les reformuler selon l'esprit et la lettre des notes d'orientation qui m'ont été fournies.

"La réflexion», est-il dit dans ces notes, «devra s'appuyer sur des recherches déjà faites, i.e. sur l'usage connu d'une méthode particulière. » Les recherches déjà faites, dans mon cals, portent sur l'architecture des raisonnements propres à l'archéologie, tels qu'on les observe dans les publications de cette discipline, les miennes y comprises. L'objectif est de mettre en lumière les mécanismes et les fondements de l'interprétation des vestiges matériels, c'est-à-dire le passage de l'observation archéologique à l'énoncé de théories ou «points de vue» relatifs aux sociétés anciennes : leurs modes de vie, les formes de leur organisation sociale, politique, économique, leurs systèmes de croyance ou de pensée, les événernents qui les ont touchées, etc. Quant à la «méthode particulière», elle est connue(?) sous le nom d'analyse logiciste: on désigne par là un mode de réécriture qui 
consiste à exprimer les constructions interprétatives sous forme de chaînes de propositions reliant les données d'observation aux «points de vue» évoqués plus haut - ou l'inverse - à la manière d'un calcul. Le paradigme computationnel est ici l'outil principal, mais il faut l'entendre dans un sens abstrait : les applications informatiques accompagnent le programme logiciste depuis trente ans, mais elles n'en sont ni l'objectif majeur ni l'inévitable prolongement ${ }^{1}$.

Je n'en dirai pas davantage sur les recherches et la méthode, et passe sans plus tarder aux questions que les initiateurs de ce recueil nous ont priés de traiter, à la lumière de nos expériences respectives.

\section{LE STATUT DES DONNÉES}

Les constructions interprétatives, en archéologie, s'appuient nécessairement sur des propositions de deux sortes: des descriptions d'objets, au sens large du terme (monuments, sites, paysages, ustensiles, etc.), et des assertions qui sont censées relever tantôt du sens commun, tantôt du savoir établi de la discipline. Ces propositions sont "déclarées» au fur et à mesure que l'auteur les mobilise pour fonder une inférence ou une dérivation particulière; et elles sont formulées dans une langue proche de ce qu'on appelle le langage «naturel », plus ou moins enrichie de termes spécialisés. De ces deux propriétés découlent les observations qui suivent, banales, mais qui intéressent directement la question du statut des données.

Des données «déclarées»: Les schématisations logicistes mettent en relief le statut particulier des propositions qui, dans une construction donnée, n'ont pas d'antécédent explicite. Ce sont elles qui constituent par définition les données de base de la construction ou, si l'on préfère, la base de données. Celle-ci est ainsi livrée sans justification articulée «en amont», du côté de l'observation empirique ou des constructions antérieures; sa raison d'être est tout entière "en aval», du côté des théories que ces données vont servir à étayer.

On ne montre ou démontre rien d'autre, ce faisant, que le statut inévitablement relatif de nos données ainsi comprises. Rien de plus banal, on l'a dit; mais la reconstitution des bases de données dans la perspective logiciste débouche sur des constats qui le sont moins. D'abord, pourquoi une re-constitution? C'est que nos pratiques de rédaction étant ce qu'elles sont, les données sont rarement énoncées comme telles, ni moins encore rassemblées d'une manière qui en facilite l'appréhension immédiate. Elles s'égrènent tout au long du texte, selon les nécessités de la présentation linéaire, sans rien qui signale clairement leur statut de données primitives dans le sens ci-dessus. Force est donc de $r e$-constituer nos bases par un travail d'analyse dont le moins qu'on puisse dire est qu'il ne va pas de soi.

À quoi servent ces reconstitutions? Essentiellement à mettre sous les yeux, c'est-àdire à l'esprit, l'ensemble des positions que nous devons accepter à la suite de tel ou tel auteur, ou au contraire reconsidérer, selon que nous épousons ou non les points de vue qu'elles servent à fonder. Les données sont regroupées de façon systématique dans des tables où apparaissent les primitifs de la construction: les objets d'étude choisis,bien entendu, mais aussi les traits distinctifs retenus (et, partant, le système de représentation sous-jacent); les analogies significatives que les uns et les autres suggèrent à l'auteur(lesquelles sont elles-mêmes, en effet, le plus souvent déclarées, intuitives et non point calculées), ainsi que les groupes (classes, types, séries, etc.) engendrés par ces analogies;

1. L'ouvrage de base est Gardin 1979; une version plus ramassée, suffisante pour notre propos présent, est Gardin 1990, où sont cités les travaux entrepris entre ces deux dates. Les apports possibles des systèmes experts en archéologie sont examinés dans Gardin et al. 1987, avec quelques exemples d'applications à l'appui. L'élargissement de ces recherches à d'autres sciences humaines est évoqué dans Gardin et al. 1981, Ennals et Gardin 1990. Enfin, un livre récent rassemble une douzaine de textes dispersés relatifs à des moments ou à des aspects particuliers du programme logiciste vu sous l'angle de ses rapports avec les sciences du discours, l'intellectique, la philosophie : Gardin 1991a. 
enfin, les présuppositions introduites au cours de l'argumentation pour alimenter le jeu des inférences ou des dérivations successives, au titre du savoir établi ou du sens commun. l'ensemble peut paraître disparate, mais nul ne saurait contester le dénominateur commun à tous ces éléments, à savoir le statut de données primitives de par leur place dans la construction.

Des données «naturelles», écrivions-nous aussi : il faut entendre par là, au minimum, que l'expression des données se fait pour l'essentiel en langage naturel; certains vont plus loin en posant que la substance des données, dans les sciences humaines, est elle-même naturelle en ce sens qu'elle s'impose à tout observateur éclairé comme une évidence irréfutable. Je ne retiendrai ici que l'acception première, minimale. Minimale, mais pourtant aussi contestable que la seconde, pour d'autres raisons : ce sont ces raisons que l'analyse logiciste fait ressortir avec force. Considérons par exemple la façon dont nous décrivons nos objets en archéologie : à première vue, rien de plus naturel, en effet, que le langage habituellement utilisé pour énoncer leurs propriétés de toute espèce: matériaux, formes, techniques de fabrication, décors, inscriptions, lieux de trouvaille, etc. Mais que l'on en vienne seulement au projet de bâtir ou d'exploiter une banque de données «naturelles » à partir de telles sources : la nécessité d'un (méta)langage documentaire apparaît aussitôt, pour établir les correspondances voulues entre des formes d'expression que l'on tient pour équivalentes, $d$ 'un certain point de vue - et il me paraît aller de soi qu'il n'est loisible à aucune science, fût-elle humaine, d'appréhender ses objets autrement que «d'un certain point de vue» (voir plus haut). Ces langages documentaires (LD) appartiennent à la famille de ce que les linguistes appelaient naguère des langues spéciales (LS), divorcées du langage naturel (LN). Le contexte dans lequel naissent et se développent ces langues spécialisées fait qu'elles présentent aussi une certaine parentí avec le langage scientifique (LS) et qu'on l'entend dans les sciences de la nature - parenté tantôt ascendante, tantôt descendante, selon que les relations logico-sémantiques du langage documentaire préfigurent une langue scientifique à venir ou qu'elles reflètent celles qui sont tenues pour établies dans une science constituée. La dynamique toujours ouverte des rapports entre ces trois familles (LN, LD, LS) a fait couler assez d'encre pour que je ne m'y attarde pas ici (bibliographie et discussion dans Gardin 1979 : 75-113). Le seul point à retenir pour mon propos présent est que nos systèmes de représentation en archéologie ne sont nullement contenus dans cette entité au demeurant assez vague que désigne le symbole $\mathrm{LN}$ : invoquez le naturel, il s'enfuit au galop.

\section{LES TECHNIQUES INFORMATIQUES}

"La question du statut des données », précise-t-on dans les recommandations aux auteurs, peut être abordée "aussi bien du point de vue des techniques informatiques que des techniques plus traditionnelles». Le biais informatique est assez apparent dans les pages qui précèdent; mais il risque de tromper. Pour être franc, je ne suis pas sûr d'apercevoir la relation entre les techniques informatiques et le statut des données. Les questions que je viens d'évoquer à ce dernier titre se posaient avant l'avènement de l'informatique; et j'ai pris soin de rappeler que l'adoption du paradigme computationnel obéissait à d'autres raisons que l'usage des ordinateurs. Ceux-ci sont d'ailleurs absents dans nombre de nos entreprises, lesquelles n'en sont pas moins toutes tournées vers «le calcul et la formalisation dans les sciences de l'homme», pour reprendre le titre d'une des premières conférences internationales sur le sujet (Jaulin 1966). Enfin et surtout, il y a plus : l'enseignement principal que je tire pour ma part de l'analyse logiciste des raisonnements a trait aux limites de la formalisation et, partant, de l'informatique elle-même, dans les recherches historiques. Je reprendrai ce point plus loin à propos du statut des explications dans nos disciplines, et $m$ 'en tiendrai ici à l'aspect technologique du sujet, comme nous y avons été invités.

L'outil informatique a été utilisé très tôt en archéologie, dès la fin des années 50; il faisait suite à l'outil mécanographique, par où j'avais commencé. Les problèmes théoriques 
restaient dans les deux cas les mêmes : quelle représentation de nos matériaux convenaitil d'utiliser pour faciliter le traitement sur machine? Une fois les «données» ainsi fixées, quelles suites d'opérations fallait-il programmer pour engendrer des «ordres» intéressants, au sens le plus large du terme -séries comparatives, typologies, sériations, réseaux, etc.? Et comment mesurer en l'espèce la validité de ces ordres - ou mieux leur «intérêt », pour nous en tenir à un critère moins sévère - en particulier dans les cas fort nombreux où l'on savait en produire plusieurs, tous bien formés, justiciables chacun d'une interprétation différente (ou plus)? Le recours à l'informatique eut, si je puis dire, l'avantage de précipiter les choses : confrontés aux multiples produits possibles du traitement formel, nous étions en quelque sorte sommés d'expliquer les raisons de nos préférences pour tel ou tel, au risque de passer alors pour d'incorrigibles bricoleurs, au même degré que les plus intuitifs de nos devanciers. Mieux ou pire encore, il nous fallait aussi expliquer les cas également nombreux où ces produits étaient en quelque sorte mort-nés : on tendait à les oublier sitôt le temps de l'«expérience» ou de l'«application-pilote» passé et le progrès des connaissances, pour autant qu'on ose ici l'évoquer, semblait n'en tenir aucun compte.

Les constats de ce genre sont devenus monnaie courante; ils suscitent l'irritation des chercheurs convaincus du contraire par leur expérience propre, assurément aussi digne de foi que la nôtre. C'est que le débat est mal posé, ou que l'on se méprend sur la portée de tels constats. Je ne songe aucunement pour ma part à bannir les «techniques informatiques» de notre arsenal méthodologique; leur utilité pratique apparaît à toutes les étapes de la recherche en archéologie - procédures d'échantillonnage, constitution et exploitation des archives de fouilles, recherches documentaires, approches heuristiques de la classification, aide à l'interprétation, etc. Ma seule réserve concerne l'équation trop vite posée entre la maîtrise de ces techniques et l'affermissement de nos constructions théoriques. Ce n'est pas déprécier l'une que de mettre en doute ses effets sur l'autre. Une manière d'illustrer l'indépendance relative des deux volets consiste à souligner leur incommensurabilité dans quelques cas concrets.

Considérons par exemple le problème de la représentation de nos objets, c'est-à-dire l'expression de leurs propriétés, lato sensu, dans des termes d'un langage particulier (LN, LD ou LS, voir $\$ 1$ ). Il fut un temps où l'on pensait que le progrès des techniques informatiques de reconnaissance des formes permettrait bientôt de remplacer les sens et le cerveau humains par des capteurs variés, couplés à un ordinateur : la rigueur formelle de la procédure informatisée était censée garantir des représentations du meilleur aloi que les descriptions «humaines», flottantes impressionistes, erratiques, etc. Nul ne tiendrait plus aujourd'hui un tel pari.

Si j'ai choisi cet exemple, c'est qu'il contient en herbe tous les éléments de la même démonstration pour d'autres types d'applications. Ainsi, l'attribution automatique d'objets à des classes préétablies peut être considérée comme un cas particulier de la reconnaissance des formes; de même, la recherche de structures dans une matrice de données, à des fins classificatoires; de même encore, la simulation des processus d'apprentissage qui président à la formation de ces classes, etc. Le fait à retenir est que la part du tuteur ou de l'arbitre humain dans chacun de ces cas de figure reste considérable. C'est là peut-être ce qui m'empêche de voir en quoi le progrès des techniques informatiques, par ailleurs admirable et bienvenu, pourrait contribuer à celui des constructions théoriques en archéologie. À moins qu'on en revienne à la vision logiciste des choses... comme je vais m'y employer maintenant.

\section{LE STATUT DE L'EXPLICATION}

À ce stade de l'exposé, le lecteur éprouve sans doute déjà une certaine répulsion à l'égard de ladite "vision": ne marque-t-elle pas, en effet, un retour à des positions scientistes que l'on croyait défuntes, en particulier dans le domaine des humanités? Telle semble être l'opinion dominante dans les discussions orales ou écrites relatives au 
programme logiciste ; les démons auxquels nous sommes alors renvoyés ont des noms familiers : positivisme, réductionisme, mécanicisme, technolâtrie, même, etc. Le passeport canadien de la revue qui $m$ 'accueille me rappelle une manifestation parmi d'autres de ce procès, à Chicoutimi, il y a une dizaine d'années. C'était au $33^{\circ}$ Congrès de l'Association canadienne-française pour l'avancement des sciences; Pierre Ouellet, qui l'organisait, m'avait aimablement invité à résumer le séminaire que je donnais au même moment à l'Université Laval sur mon sujet d'aujourd'hui, lequel paraissait entrer assez bien dans le champ couvert par le congrès : «les discours du savoir». Le résultat fut désastreux. Le débat qui suivit porta tout entier sur l'infection scientiste dont je devais être victime, mais à aucun moment sur les questions d'épistémologie dont j'avais parlé. Pourtant, lorsque je retourne aujourd'hui au volume des Actes (Ouellet 1986), je suis frappé par l'étonnante parenté entre les titres donnés aux différentes parties du livre et les thèmes qui n'ont cessé de $m^{\prime}$ 'occuper sous couvert du programme logiciste (les références qui suivent sont aux chapitres d'une anthologie récente: Gardin 1991) : «l'analyse du discours scientifique » (chap. 4), «Science et linguistique» (chap. 8), «Science et littérature» (chap. 9), «L'analyse des discours "savants" " (chap. 12), "Sémiotique et philosophie du savoir» (chap. 10 et 3, respectivement). Dès lors, comment se fait-il que le dialogue n'ait pu s'établir à Chicoutimi, ni ailleurs en d'autres occasions semblables? Les insuffisances ou les maladresses de ma présentation ne sont, je crois, qu'une partie de l'explication. L'autre tient au Zeitgeist qui semble aujourd'hui prévaloir dans les sciences de l'homme, comme obsédées par les dévoiements qui les menacent sous l'effet de tels ou tels biais idéologiques, et par le devcir d'au moins les reconnaître faute de savoir toujours y résister. L'idéalisation de la science est l'un de ces biais, parmi les plus attaqués : l'engouement pour la technologie en est un autre, apparenté, qui suscite les mêmes rejets. Or le programme logiciste, à peine entendu, est aussitôt classé comme une manifestation de plus d'un scientisme étroit, aggravé par le culte de l'ordinateur: on comprend dans ces conditions qu'il n'intéresse guère les chercheurs acquis à des positions plus subtiles, sous le couvert de disciplines ou d'écoles bien établies - sociologie de la connaissance, "psychologie populaire», anthropologie cognitive, pragmatique, herméneutique, sémiotique, école de Francfort et sans doute bien d'autres encore, dans les larges avenues de la science «postmoderne» des faits humains.

En rassemblant ainsi sous une même étiquette au demeurant mal définie, tant d'approches différentes, je risque de simplifier indûment ce qui oppose chacune à l'approche logiciste. L'amalgame me paraît pourtant justifié au moins pour ce qui concerne la question posée dans le présent paragraphe, sur le statut de l'explication. Le contraste est en effet massif entre deux familles de réponses : celles de la science «en général », à laquelle le logicisme n'ajoute rien, et celles du postmodernisme, toutes tendances confondues. Dans le premier cas, la valeur explicative des (re)constructions s'apprécie selon les critères habituels en science : cohérence des opérations mises en jeu, correspondance entre les résultats théoriques et les observations empiriques du moment. Dans le second cas, ces critères sont jugés tantôt impraticables, tantôt indésirables, s'agissant de phénomènes ou d'objets humains dont on pense qu'ils ne se laissent pas «expliquer» de la même manière que le mouvement des astres ou la structure de la matière; et l'on propose d'asseoir sur des bases psycho-sociales ou culturelles inévitablement locales, dans l'espace comme dans le temps, la valeur cognitive de nos constructions.

L'erreur commune est de considérer que le but, avoué ou non, de l'analyse logiciste, est de rehausser le statut des explications du premier type, scientifiques dans l'acception que l'on dit étroite du terme, en mettant l'accent sur toutes les raisons que l'on a de dénier cette qualité aux écrits du second type. Cette vision hiérarchique des choses n'est pas la mienne;j'ai pris très tôt la précaution de souligner que je n'avais pour la Science avec une lettre majuscule aucune admiration exclusive, au point de rejeter a priori toute autre manière de discourir des faits humains. Bien plus, la Littérature avec une lettre majuscule, qui est une de ces manières (à ne pas confondre avec la prose des sciences de l'Homme), a selon moi des vertus cognitives au moins égales: je l'ai écrit si souvent que je n'ose plus 
donner les références à ces textes déjà répétitifs. La chose curieuse est que, semble-t-il, le message ne passe pas : cette apologie de la Littérature mêlée à un programme de recherche attaché aux valeurs de la Science paraît à la plupart suspecte - boutade, coquetterie, pirouette, voire incohérence pure et simple, a-t-on écrit. Fort heureusement, cette coexistence nécessaire des deux genres, mieux encore leur inévitable complémentarité dans les travaux historiques, pour ne parler que de ceux-là, constituent aujourd'hui des thèmes que l'on aborde enfin sous l'angle technique, et non plus idéologique. C'est sur ce seul terrain que je souhaite traiter la question qui nous est posée, du statut de l'explication en sciences sociales; mais mon argumentation sera plus facile à suivre si je réponds d'abord à cette autre, inscrite entre guillemets dans nos instructions, «la question des rapports entre objet, méthode, théorie, pratique et sujet de l'action».

\section{OBJETS ET SUJET, THÉORIES ET PRATIQUE, ETC.}

L'idée saugrenue m'est d'abord venue de considérer dans la phrase précitée toutes les paires de termes dont nous étions invités à commenter les rapports : l'exercice n'est pas stérile, mais il aboutit sous ma plume à des propositions répétitives, d'une paire à l'autre, qui le rendent ennuyeux. J'ai donc essayé de le réduire en m'arrêtant aux trois cas de figure suivants : le sujet et le reste, la théorie et la pratique, la méthode et le reste.

Le sujet et le reste - «Le retour en force du sujet » est un des thèmes caractéristiques du Zeitgeist que j'évoquais plus haut; il s'entend à travers mille variations dans les œuvres consacrées à la spécificité des sciences de l'Homme, mais aussi bien dans une littérature plus vaste touchant le mouvement des sciences en général, nature et culture confondues. Mon opinion en la matière n'importe guère ici; la seule question pertinente, dans le cas présent, me semble être celle-ci : est-il possible de bâtir des théories relatives aux objets qui nous occupent, en archéologie (je reviens pour un temps à ma seule discipline), d'une manière qui prenne en compte les points de vue des «sujets»? Il est entendu qu'il faut comprendre sous ce terme non seulement les acteurs de l'histoire auxquels nos objets permettent bon an mal an de remonter, mais aussi nous-mêmes, archéologues, qui pratiquons ces remontages avec nos biais culturels propres. Ma réponse à la question n'est pas la même dans les deux cas. La reconstitution de l'univers psychique des gens d'autrefois - mentalités, cosmogonies, symbolique, etc. - est un exercice que les historiens ont pratiqué bien avant que les apôtres d'une archéologie (post-)moderne en aient fait un impératif moral. Les travaux de cette nature, d'un côté comme de l'autre, se prêtent à l'analyse logiciste au même titre que toute espèce de construction interprétative; et les leçons qu'on en tire ne diffèrent pas fondamentalement de celles que j'ai déjà indiquées plus haut. La particularité du genre tient à la présence d'inférences qui mettent en jeu, dans les formules de réécriture (partie gauche et/ou partie droite, selon les cas), des propositions plus difficiles à «vérifier» que d'autres, touchant les représentations mentales attribuées à des sujets qui ne sont plus là pour les authentifier. L'historien découvre parfois dans les textes des témoignages qui renforcent ses restitutions, fût-ce sur le seul axe de la cohérence conceptuelle à défaut de correspondance empirique; malheureusement, les objets qu'étudie l'archéologue sont à cet égard moins suggestifs, pour ne pas dire irrévocablement ambigus. Quoi qu'il en soit, le point important n'est à mon avis pas là : le principe même d'une "vérification » n'est-il pas proscrit dès lors qu'une théorie s'adresse à des représentations que les sujets porteurs peuvent n'avoir jamais éprouvées ni conçues? Je ne fais ici que reprendre un argument souvent invoqué dans les écoles post-modernes, où les voies de l'interprétation s'apparentent à celles de la psychanalyse peu portée à faire de ses patients les arbitres de sa valeur scientifique. Dans ce cas, toute proposition relative à l'univers psychique de nos sujets - morts ou vivants - me paraît vouée à l'indécidabilité, tout chercheur étant libre de (se) le représenter de la manière qui lui paraît la plus... juste, intéressante, convenable, stimulante? A quoi je ne trouve au demeurant rien à redire, une fois admis que l'on bascule dès lors dans un univers de discours où la valeur des théories se mesure à des effets sociaux parfaitement estimables, 
dans les deux sens du terme, mais qu'il est difficile d'ordonner les uns par rapport aux autres pour déceler, en matière humaine, un «progrès des connaissances » analogue à celui dont les sciences de la nature nous ont donné l'habitude ou le goût. Qu'il soit en revanche possible de mettre à nu la subjectivité des interprètes que nous sommes dans l'exercice de notre métier ne me paraît pas douteux (Gardin 1991b). Tel est précisément l'un des buts de l'analyse logiciste appliquée aux argumentations savantes :l'élucidation des prémisses sur lesquelles reposent les inférences dont elles sont faites est au cœur de l'entreprise. Soit, rne direz-vous, mais n'est-ce pas aussi le propre des études qui prennent la science pour objet à d'autres titres - sociologie des sciences, histoire des sciences, psychologie des sciences, philosophie des sciences? On me saura gré de ne pas répéter les raisons maintes fois données de distinguer nos visées respectives : les nôtres ne sont pas d'expliquer les théories concurrentes et leur accumulation dans les sciences de l'homme en les rapportant à des circonstances extrinsèques - sociologiques, historiques, etc. - mais plutôt de les décrire dans des termes qui fassent mieux ressortir l'architecture et les fondations comparées de chacune, à des fins qui ne dépassent pas celles d'une épistémologie résolument «pratique».

Pratique et théorie - Je m'empare aussitôt de ce dernier terme pour introduire l'idée que je me fais, sur des rapports entre pratique et théorie dans la méthodologie des sciences sociales, comme on nous y invite. J'appelle «pratique» une épistémologie inséparable de l'exercice d'une activité scientifique particulière, spécialisée, inscrite dans un champ nécessairement limité, à la différence de l'épistémologie spéculative et générale des philosophes - honneur et grâces leur soient rendues. Cette épistémologie pratique soulève des questions qui s'adressent aux spécialistes du domaine où elle choisit de s'exercer, c'est-à-dire à des praticiens de la recherche, comme son nom l'indique, plutôt qu'à cles théoriciens généralistes. Un archéologue ne saurait résoudre les problèmes d'épistémologie pratique posés par un sociologue, sauf s'ils intéressent la partie commune de la logique interprétative à laquelle l'un et l'autre font appel en la qualifiant dès lors de «naturelle» ou en l'assimilant au «sens commun». J'ai dit ailleurs et plus d'une fois les doutes que cette référence à la communauté naturelle de nos vues m'inspirait dans le contexte qui est le nôtre, la recherche scientifique : le fait que celle-ci s'applique à des objets humains ne saurait prévenir le caractère inévitablement artificiel et singulier de nos théories, puisque ce sont encore et toujours des constructions et qu'elles sont en principe le fruit de raisonnements suffisamment hors du commun pour valoir aux auteurschercheurs un statut distinct.

Théorie et pratique sont ainsi liées de la façon la plus intime qui soit: l'analyse logiciste ne s'intéresse à l'une qu'à raison de ses reflets dans l'autre. Rien de plus banal, me dira-t-on, s'agissant d'une discipline empirique comme l'archéologie; j'en conviens, mais les sciences sociales ne sont-elles pas dans le même cas? Et que peut-on dire dès lors du rapport entre pratique et théorie qui ne l'ait pas été mille fois à propos des sciences d'observation en général?

Certains répondront sans doute qu'une façon de renouveler ce débat est de réaffirmer l'originalité des sciences humaines, qui tendrait à cette liberté qu'elles se donnent de séparer les deux modes d'action, théorique et pratique, sans mettre en cause leur statut scientifique. L'archéologie postmoderne - encore elle - est à cet égard exemplaire : elle foisonne de constructions de la plus haute volée relatives aux hommes d'autrefois, à leurs croyances, à leurs institutions, à leurs mondes imaginaires, bâties à partir de nos bric-àbrac habituels; mais l'analyse des raisonnements qui relie ceci à cela montre clairement que les propositions supérieures - je veux dire les conclusions atteintes au terme de l'argurnentation - sont fondées sur des pétitions de principe que l'observation empirique ne permet pas de vérifier(ou de falsifier si l'on préfère cette version de la même affaire). Nous sommes donc bien en présence de théories coupées de la pratique : les points de vue ainsi formés ne sauraient passer pour des hypothèses de travail, puisqu'on est incapable de concevoir le genre de «travail» auquel il faudrait se livrer sur le terrain pour les étayer. Le divorce s'exprime d'ailleurs de façon réciproque : l'archéologie de terrain et la part de 
savoir, qu'il faut bien qualifier d'établi, à laquelle se réfèrent les livres d'histoire qui en sont tributaires paraissent progresser sans dommage à l'écart des constructions de l'espèce postmoderne.

Est-ce à dire qu'il faille proscrire celles-ci? Parlons plus clair : l'analyse logiciste condamne-t-elle les constructions de cette espèce? En aucune façon: nous ne faisons qu'ajouter au débat philosophique une approche technique de leur «différence» (avec un a, le cas échéant), qui nous paraît compléter utilement le dossier. Le rapport entre pratique et théorie est l'un des lieux où se joue cette différence; une voie connexe est celle de la validation. On sait les embarras qu'elle cause à la rationalité «alternative» des sciences sociales, à en juger par le nombre de livres ou d'articles qui lui sont chaque année consacrés. L'archéologie n'échappe pas à la règle; une de ses dernières contributions au sujet mérite ici mention: "How then, asks the positivist-trained archaeologist, do we validate our hypotheses? [...] Well, one answer is that we don't ... There can only be continual debate and approximation» (Hodder 1986:93-4). La réponse logiciste à la question n'est certes pas celle-là, mais elle n'est pas non plus celle du positivisme : nous acceptons tous les critères de validation que l'on voudra, y compris les critères psychologiques ou sociaux de l'herméneutique, à condition qu'ils soient explicitement déclarés comme une des composantes des théories proposées; et nous nous interrogeons sur le statut scientifique (ou autre) des constructions qui entendent échapper à cette obligation.

La méthode et le reste - Quels rapports la méthode entretient-elle avec les autres termes de la question qui nous est posée : l'objet, la théorie, la pratique, le sujet? Les observations qui précèdent laissent deviner la réponse logiciste; je la résumerai en trois points. (a) Les seules méthodes qui nous occupent sont celles de l'argumentation, telles qu'elles se manifestent dans les constructions interprétatives qui ont été jugées dignes d'une publication dans la littérature spécialisée. Nous n'avons donc rien d'original à dire sur les questions de méthodologie soulevées à propos d'autres moments ou d'autres outils de la recherche dans les sciences sociales. (b)Toutes les constructions sont à cet égard bonnes à prendre pour peu qu'elles aient l'oreille d'une fraction raisonnable du corps des chercheurs, sans que nous en excluions a priori aucune. Il s'ensuit que toutes les méthodes de raisonnement pratiquées dans ces constructions ont à leur tour le même intérêt pour notre propos, indépendamment des écoles de pensée auxquelles on les rattache. Bref, je souscrirais volontiers en la matière à l'aphorisme célèbre de Feyerabend (déjà invoqué dans Gardin 1979, en quatrième de couverture) : «anything goes 》- d'où la brièveté du présent paragraphe. (c) Avec quelques nuances, cependant :tout est bon à considérer, dans les univers du discours qui sont les nôtres, mais tous les modes de pensée ne sont peut-être pas également bons à retenir après le passage de l'analyse logiciste. C'est à ce stade que nos critiques agitent le spectre du positivisme, lorsque, de descriptive qu'elle était, l'analyse devient à leurs yeux normative, en paraissant prôner un retour à la science pure et dure qui excommunierait la quasi-totalité de nos écrits dans les humanités.

La réalité est autre; c'est par elle que je conclurai, en répondant du même coup à «la question centrale» du scénario qui nous a été proposé, dans les termes suivants :

\section{L'ANALYSE DES LIMITES ET POSSIBILITÉS DES TECHNIQUES UTILISÉES}

Les techniques utilisées sont ici celles du calcul, qui comprend l'informatique mais aussi bien la dépasse, comme je m'en suis expliqué eu début de cette note. Quant aux «limites et possibilités» de la chose, sur lesquelles on nous interroge, je les résumerais volontiers par une pirouette, en reliant ainsi les deux termes : les possibilités du calcul (à mes yeux les plus intéressantes) tiennent à la mise en évidence de ses limites dans nos discussions. L'addition entre parenthèses est là pour prévenir les protestations de chercheurs plus hardis que moi dans l'appréciation des mérites de l'ordinateur et de la mesure - numérique - dans les sciences de l'homme (Genêt 1992). Un second complément est nécessaire : baliser les limites du rationnel calculable est une chose, envisager la ou les conduites possibles au-delà de ces limites en est une autre, qui fait aussi partie selon moi 
de l'enquête logiciste. Celle-ci atteint donc ses fins lorsqu'elle force la réflexion, voire parfois l'action, sur deux thèmes qui n'ont ni l'un ni l'autre rien à voir avec le positivisme : (a) en premier lieu, la recherche de lignes de démarcation plus claire entre la part de nos raisonnements qui ressortit au mode «logico-scientifique», comme l'appelle J. Bruner (1986 : 12), et une part complémentaire, alternative, résiduelle sur la nature ou le mode de laquelle il n'est pas nécessaire $\dot{a}$ ce stade d'émettre aucun jugement; (b) dans une seconde étape, la recherche d'une spécificité de cet autre mode, de quelque façon qu'on le nomme, dans l'idée qu'il faut bien lui en trouver une pour justifier le statut de chercheur ou d'enseignant dans ce que nous continuons d'appeler les sciences de l'homme.

Les limites et les possibilités du logicisme, en dernière analyse, sont celles que chacun reconnaît à ce double mouvement, selon son expérience ou sa philosophie. Un mouvement qu'il faut cependant considérer dans la perspective longue de l'histoire des sciences : au-delà des appréciations présentes de quelques-uns, dans un sens ou dans l'autre, l'avenir du logicisme me paraît se confondre avec celui des sciences humaines elles-mêmes, dans l'«entre-deux» (Passeron 1991) où celles-ci entendent aujourd'hui légitimer leur place. J'ai montré ailleurs comment l'enquête logiciste débouchait inévitablement sur des questions relatives à la définition de cette «troisième voie» de la connaissance qui ne serait, pour les uns, ni celle de la science ni celle de la littérature; pour d'autres, ni celle des constructions symboliques divorcées des logiques et langages «naturels », ni pourtant tout à fait celle de l'argumentation courante dans la vie quotidienne jusqu'au café du commerce, ironisait naguère Cibois (1989: 73). Il est ennuyeux de se répéter : je finirai donc comme j'ai commencé, en suggérant au lecteur que ces questions intéressent un examen critique des références de la note 1.

Jean-Claude GARDIN Centre national de la recherche scientifique Unité propre de recherche no 315 27, rue Damesme 75013 Paris, France 


\section{ANNEXE}

Texte de l'Appel d'offres diffusé par la Délégation Générale à la Recherche Scientifique et Technique en 1973 et 1974 au titre d'un programme intitulé «Informatique et sciences humaines»

L'Action Complémentaire Coordonnée «Informatique et Sciences Humaines» se propose d'explorer les problèmes méthodologiques posés par l'application actuelle ou souhaitable de l'informatique aux sciences humaines. Un effort de réflexion est en effet nécessaire moins pour ajouter à la diversité des méthodes actuelles que pour les clarifier. La liste des thèmes de recherche proposés ci-dessous ne constitue pas un programme rigide et l'on accueillera avec intérêt toute autre problématique proposée dans le même esprit.

\section{THĖMES DE RECHERCHE PROPOSÉS}

\section{A) Constitution des données}

I- Qu'est-ce qu'une donnée en sciences humaines - qu'elle soit d'observation textuelle ou perceptuelle - par rapport à un objectif et dans un champ déterminés? Procédures de constitution, stratégies de sélection des données.

II- De l'observation aux langages : la correspondance objet-langage et la critique des langages de désignation :

- de l'observation à une représentation linguistique, naturelle ou formalisée, tique.

- de la représentation linguistique naturelle à un langage formalisé pour traitement informa-

III- Extraction et exploitation des données textuelles : analyse de textes et théories linguistiques. Étude de leur relation, possibilités d'un système conceptuel commun.

B) Contrôle et traitement des données

I - Contrôle : cohérence interne et cohérence croisée des données :

- optimisation des contrôles en coût-efficacité

- méthodes générales de traitement des contrôles

- méthodes d'apurement.

II-Traitement : les méthodes, leur fondement, leur justification. Des présupposés des choix à la critique des résultats.

1. Critique des hypothèses implicites dans l'utilisation des techniques; analyse de régression, analyses factorielles et leurs variantes, segmentation, classifications dites automatiques, etc.

2. Méthodes spécifiques d'analyse mathématique et statistique de séries (temporelles notamment) à valeurs non numériques; analyse de séries multidimentionnelles.

3. Analyse, ses implications : critères de choix entre une analyse réduite, impliquant une perte d'information, et une analyse étendue qui accroît les difficultés d'exploitation.

III-Méthodes générales de validation. Procédures de confrontation des résultats du calcul et des données expérimentales.

C) Systèmes de données

I- Rationalisation des systèmes de traitement et de réseaux de données en sciences humaines : formats d'entrée, modes de manipulation, reformatage, etc.

II-Détermination des identifiants communs à plusieurs systèmes. Intégration de fichiers.

III- Problèmes de masse critique : solutions spécifiques imposées par la taille des systèmes de données en sciences humaines.

D) Position de l'informatique dans les sciences humaines

Statut épistémologique, pédagogique, sociologique.

I- Informatique et disciplines des sciences humaines :rapports, subordination relative.

II- L'informatique dans l'enseignement des sciences humaines : techniques auxiliaire, ferment critique.

III-Aspects sociologiques de l'insertion de l'informatique dans les sciences humaines. 


\section{RÉSUMÉ}

Dans une perspective logiciste, les textes interprétatifs des sciences sociales sont justiciables d'une réécriture à la manière d'un calcul, qui fait ressortir les données et les opérations constitutives du raisonnement. Les recherches menées dans cette voie, en archéologie notamment, éclairent d'un jour nouveau les mécanismes et les fondements des constructions propres aux sciences de l'homme. Les leçons tirées portent sur le statut de l'explication dans ces disciplines, sur la part du sujet dans la conception et dans la validation de nos théories, sur l'inlérêt d'une épistémologie pratique où ces théories sont jaugées selon des critères de valeur clairement déclaré:, sur la neutralité d'une telle épistémologie à l'égard des méthodes des sciences sociales; enfin et surtout, sur la fragilité d'un entre-deux où celles-ci tentent sans relâche de se placer, entre science et littérature ou sens commun.

\section{SUMMARY}

From the point of view of logic, interpretive texts in the social sciences are liable to being rewritten as computation, emphasizing the data and operations which make up the reasoning process. Research carried out in this perspective, particularly in archeology, throws new light on the mechanisms and foundations of constructions specific to the human sciences. The lessons learned relate to the status of explication in these disciplines, the part played by the subject in the conception and validation of theories, the interest of practical epistemology by which these theories are gauged using clearly defined value criteria, the neutrality of such an epistemology in relation to methods in the social sciences and, finally and most importantly, the fragility of the area in between where the social sciences are continually attempting to find their place, between science and literature or common sense.

\section{RESUMEN}

En una perspectiva lógica, los textos interpretativos de las ciencias sociales caen bajo la juridicción de una re-escrilura a la manera de un cálculo, la cual hace resaltar los datos y las operaciones constitutivas del razonamiento. Las investigaciones llevadas a cabo por esta vía, en arqueología en particular, dan nuevas pistas sobre los mecanismos y los fundamentos de las construcciones propias a las ciencias del hombre. Las lecciones sacadas se refieren al estatus de la explicación dentro de estas disciplinas, al lugar ocupado por el sujeto en la concepción y en la validación de nuestras teorías, al interés de una epistemología práctica en la cual estas teorías son establecidas según criterios cuyo valor ha sido claramente enunciado, a la neutralidad de tal epistemología en relación a los métodos de ciencias sociales; finalmente y por sobre todo, a la fragilidad de la posición intermedia en la cual éstas tienden incansablemente a colocarse, entre ciencia y literatura o sentido común.

\section{BIBLIOGRAPHIE}

CIBOIS, Ph. (1989), «Pour une science sociale synchronique, "La Revue du MAUSS, $\mathrm{n}^{\circ} 4$ (nouvelle série), pp. $70-84$.

BRUNER, J. (1986), Actual Minds, Possible Worlds, Harvard University Press, Cambridge, Mass.

ENNALS, R. \& GARDIN, J.-C. (eds.), (1990) Interpretation in the Humanities : Perspectives from Artificial Intelligence, The British Library, London.

GARDIN, J.-C. Une archéologie théorique, Hachette, Paris. 1979 Adaptation française de Archaeological Constructs : an Aspect of Archaeological Theory, Cambridge University Press et Éditions de la Maison des Sciences de l'Homme, Cambridge-Paris, 1980.

(1990) «The Structure of Archaeological Theories » in A. Voorrips (ed.), Mathematics and Information Science in Archaeology : a Flexible Framework, Holos, Bonn, pp. 7-28.

(1991a) Le Calcul et la Raison : essais sur la formalisation du discours savant, Éditions de l'École des Hautes E.tudes en Sciences Sociales, Paris.

(1991b) Le rôle du sujet dans les sciences de l'homme : essais d'évaluation objective. Revue Européenne des Sciences Sociales, XXIX, no 89, pp. 91-102.

GARDIN, J.-C. et al. (1981) (avec M.-S. LAGRANGE, J.-M. MARTIN, J. MOLINO, J. NATALI), La logique du plausible: essais d'épistémologie pratique (en sciences humaines). Éditions de la Maison des Sciences de l'Homme, Paris, $1987,2^{\mathrm{e}}$ édition revue et augmentée.

1987 (avec O. GUILLAUME, P. HERMAN, A. HESNARD, M.-S. LAGRANGE, M. RENAUD et E. ZADOR.A-RIO), Systèmes experts et sciences humaines : le cas de l'archéologie. Eyrolles, Paris. Version anglaise : Artificial Intelligence and Expert Systems : Case Studies in the knowledge Domain of Archacology, Ellis Horwood, Chichester 1988.

GENET, J.-P. (1992), « Calcul ou logicisme ? A propos d'un ouvrage récent » [J.-C. Gardin 1991 a ci-dessus] in Histoire \& Mesure, VII, 1/2, pp. 17 I-179.

JAULIN, B. (éd.) (1966) Calcul et formalisation dans les sciences de l'homme. Paris, Éditions du CNRS. 
OUELLET, P., avec la collaboration de Khadiyatoulah Fall (eds.), (1986) Le discours du savoir, Actes du colloque tenu à l'université du Québec à Chicoutimi lors du 53' Congrès de l'AFCAS du 20 au 24 mai 1985, Cahiers de l'AFCAS n 40, Presses de l'Université du Québec, Sillery.

PASSERON, J..C. (1991), Le raisonnement sociologique : l'espace non-poprerien du raisonnement naturel, Paris, Nathan. 